\title{
The Digitized First Byurakan Survey (DFBS) as UNESCO Documentary Heritage
}

\author{
A. M. Mickaelian ${ }^{* 1}$, L. A. Sargsyan ${ }^{1}$, G. A. Mikayelyan ${ }^{1}$, K. S. Gigoyan ${ }^{1}$, R. Nesci ${ }^{2}$, and C. Rossi ${ }^{2}$ \\ ${ }^{1}$ NAS RA V. Ambartsumian Byurakan Astrophysical Observatory (BAO), Byurakan 0213, Aragatzotn province, Armenia \\ ${ }^{2}$ Sapienza Università di Roma, Italy
}

\begin{abstract}
The famous Markarian Survey (or the First Byurakan Survey, FBS) was carried out in 1965-1980. Its 2000 plates were digitized in 2002-2007 and the Digitized FBS (DFBS, www.aras.am/Dfbs/dfbs.html) was created. New science projects have been conducted based on this low-dispersion spectroscopic material. The Armenian Virtual Observatory (ArVO, www.aras.am/Arvo/arvo.htm) database accommodates all new data. The project was conducted in collaboration with the Italian, USA and German scientists, as well as the Armenian Institute of Informatics and Automation Problems (IIAP) and continued during 6 years in 2002-2007. Markarian Survey and its digitized version were included in UNESCO Documentary Heritage "Memory of the World" International Register in 2011 as one of the rare heritage items from science.
\end{abstract}

Keywords: photographic plates - astronomical plate archives - Markarian Survey - digitization - astrometry - photometry - spectroscopy - databases - Virtual Observatories.

\section{Introduction}

Markarian survey (or the First Byurakan Survey, FBS) carried out by B. E. Markarian, V. A. Lipovetski, and J. A. Stepanian in 1965-1980 with BAO 1m Schmidt telescope and $1.5^{\circ}$ prism, was one of the most efficient and important survey in astronomy. It was the first systematic objective-prism survey, the largest objective-prism survey of the Northern sky (17,000 sq. deg) and it was a new method of search for Active Galactic Nuclei (AGN). There are a total of $\sim 40,000,000$ spectra for $\sim 20,000,000$ objects in the entire survey (Markarian et al., 1989). The original aim was the search for galaxies with UV excess (Markarian et al., 1989, Mazzarella \& Balzano, 1986). It resulted in discovery of 1515 UV-excess (UVX) galaxies, including more than 200 AGN and more than 100 SB galaxies. Markarian survey led to the classification of Seyferts into Sy1 and Sy2 (Weedman \& Khachikyan, 1968), the definition of Starburst galaxies (Weedman, 1977), and several other projects, such as FBS Blue Stellar Objects (BSOs, Mickaelian 2008), late-type stars (Gigoyan \& Mickaelian, 2012), optical identifications of IRAS sources (Byurakan-IRAS Galaxies (Mickaelian \& Sargsyan, 2004) and Byurakan-IRAS Stars (Mickaelian \& Gigoyan, 2006), BIG and BIS objects, respectively).

The Second Byurakan Survey (SBS) was also carried out with BAO $1 \mathrm{~m}$ Schmidt and was the continuation of FBS to fainter magnitudes (Stepanian, 2005). FBS is now digitized and the Digitized First Byurakan Survey (DFBS, Massaro et al., 2008, Mickaelian et al., 2007) is available online. It provides 40,000,000 spectra for 20,000,000 objects at high Galactic latitudes. Detailed description of FBS, SBS and DFBS is given in Mickaelian (2016).

The huge amount of spectral information contained in the FBS plates allowed the development of several other projects based on the FBS, the most important is the discovery and investigation of blue stellar objects (Mickaelian, 2008), a survey for late-type stars (Gigoyan et al., 2003), and the optical identifications of sources from the IRAS catalogue. The sample of stellar objects is available at CDS (Byurakan-IRAS Stars, BIS; Mickaelian \& Gigoyan, 2006), and a similar catalogue for IRAS galaxies (Byurakan-IRAS Galaxies, BIG; Mickaelian \& Sargsyan, 2004) will soon appear at CDS. The number and classes of new objects discovered within the FBS made clear the need for open access to this information by the entire astronomical community.

*aregmick@yahoo.com, Corresponding author 


\section{Digitized First Byurakan Survey - DFBS}

A number of digitization projects have been accomplished at BAO, including the most important one, Digitized First Byurakan Survey (DFBS; http://www.aras.am/Dfbs/dfbs.html; Massaro et al., 2008, Mickaelian et al., 2007) based on the digitization of the famous Markarian Survey (Markarian et al., 1989). It consisted of a number of steps:

- Development of technical principles of the Project, necessary Equipment

- Collection of all photographic plates of the Markarian Survey

- Revision and accounting of the plates and observing journals

- Scanning of a few dozens of plates to set up the necessary parameters for the scanning of all plates

- Input of data from observing journals; Creation of the DFBS Database (Mickaelian et al., 2006b)

- Creation of the DFBS Webpage and User Interface

- Scanning of photographic plates

- Archiving on HDDs and DVDs

- Astrometric plate solution; Extraction of images and spectra; Wavelength calibration; Density and flux calibration; Multiband (UBVR and POSS O/E) photometry

- Making up template low-dispersion spectra; Numerical classification of low-dispersion spectra

- DFBS catalogue and database, web page and user interface

- Visualization of DFBS on the sky map; creation of electronic interactive DFBS map and search system

- Proposing and discussing new research projects

The spectra extraction and analysis software is described in Mickaelian et al. (2010) and Knyazyan et al. (2011). DFBS plate database is available in Vizier, Strasbourg (Mickaelian et al., 2005a).

The DFBS project is relevant for preserving a very large database that was a milestone in the history of astronomy and to offer to the scientific community a new tool for investigating the properties of the nearby universe. The participating teams were: Byurakan Astrophysical Observatory (BAO, Armenia), Dipartimento di Fisica, 'La Sapienza' Università di Roma (Italy), Cornell University (Ithaca, NY, USA), MIGG Informatica \& Ricerca (Italy), Hamburger Sternwarte (HS, Germany), and the Institute for Informatics and Automation Problems (IIAP, Yerevan, Armenia).

Digitization of the FBS plates started in 2002. After several test scans on a set of plates, all of the FBS plates were digitized with an Epson Expression 1680 Pro scanner at the Byurakan observatory. DFBS was the first digitization project conducted by scanner in Armenia, and its results allowed a number of other similar projects. The scanning resolution was $1600 \mathrm{dpi}$, so the pixel size is about 16 microns. The typical length of an FBS spectrum is $\sim 1.7 \mathrm{~mm}$, which gives 107 pixels along the wavelength direction. An 'ad hoc' program SCANFITS written by Stefano Mottola (DLR - Institute of Planetary Research, Berlin) allows the resulting image to be written directly in FITS format with corresponding information about the plate in the header. All 1874 plates in 1139 fields (Mickaelian et al., 2005b) were digitized and stored on DVDs.

The next steps were the astrometric (plate) solution (obtained with an accuracy of $\sim 1$ " rms or 0.6 pixel), automatic extraction and classification of the spectral data in a DFBS image, and wavelength calibration (only an approximate wavelength calibration scale was obtained because the dispersion is strongly non-linear, from about $22 \AA /$ pixel at the blue edge of the spectrum to about $60 \AA /$ pixel at the red edge, with mean dispersion $32.7 \AA$ /pixel and dispersion of about $28.5 \AA /$ pixel at $\mathrm{H} \gamma$ ).

Its main scanning and resulting features are given in Table 1.

The DFBS database presently is stored on a dedicated server at the 'La Sapienza' University in Rome and can be accessed through a web interface. Mirror sites are available in Yerevan at the IIAP, Trieste (Italy) and Observatoire de Paris (France). The database includes all the digitized plates and the automatically extracted spectra. Only for F-type plates (IIaF, IIAF, IIF, and 103aF) the automatic procedure do give reliable results, and for such plates approximate $\mathrm{B}$ and $\mathrm{R}$ magnitudes have also been computed and are 

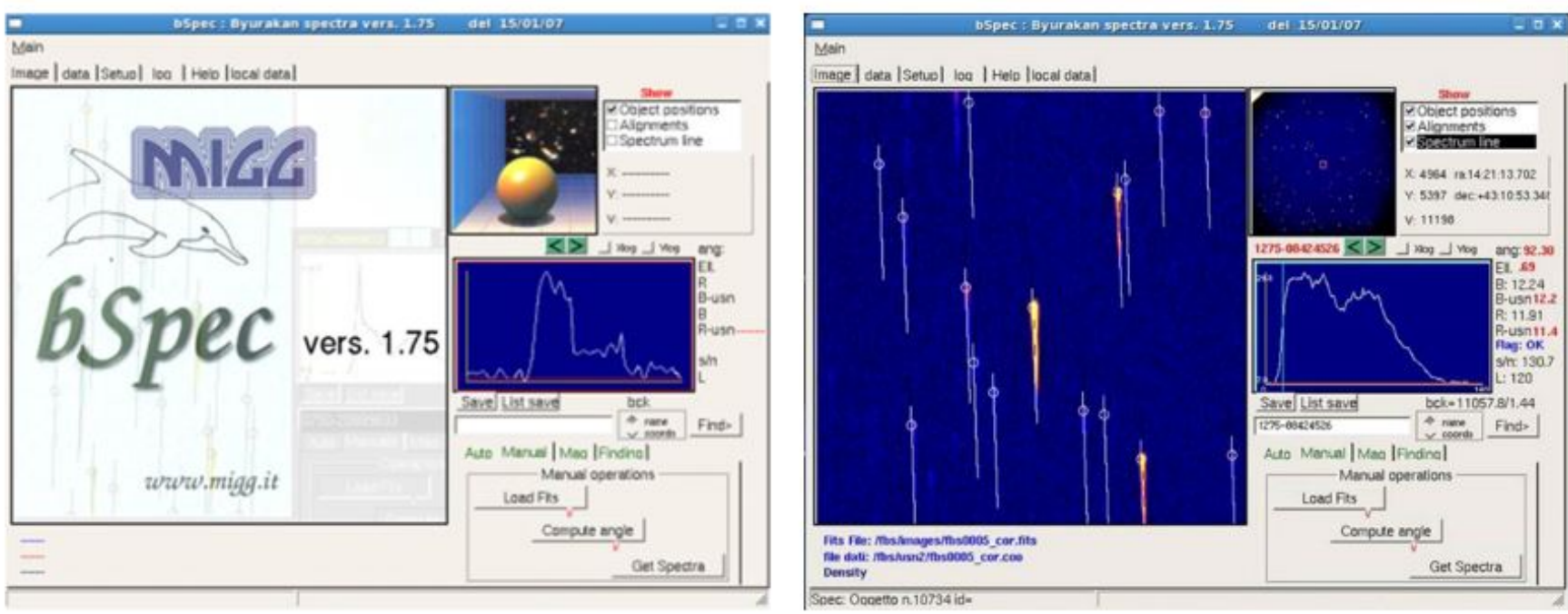

Figure 1. Dedicated software bSpec for the DFBS images and spectra extraction and analysis, an example of spectra extraction.

Table 1. Main scanning and resulting characteristics of the DFBS.

\begin{tabular}{|l|l|}
\hline Items & Description \\
\hline Teams & Byurakan Astrophys. Obs., Univ. Roma "La Sapienza", Cornell Univ. \\
\hline Years & $2002-2005$ \\
\hline Instrument & Epson Expression 1680 Pro scanner \\
\hline Scanning options & 1600 dpi $(15.875 \mu$ pix size $), 16$ bit, transparency mode, "scanfits" \\
\hline Plate size & $9601 \times 9601$ pix, $176 \mathrm{MB}$ file \\
\hline Spectra & $107 \times 5$ pix $(1700 \mu$ in length $)$ \\
\hline Dispersion & $33 \AA /$ pix average $(22-60 \AA /$ pix $), 28.5$ at $\mathrm{H} \gamma$ \\
\hline Spectral resolution & $50 \AA($ average $)$ \\
\hline Astrometric solution & $1 "$ rms accuracy \\
\hline Scale & $1.542 " /$ pix \\
\hline Photometry & $0.3^{m}$ accuracy \\
\hline Data volume & 1874 plates, $\sim 400 \mathrm{~GB}$ \\
\hline Number of objects & $\sim 20,000,000(\sim 40,000,000$ spectra $)$ \\
\hline
\end{tabular}

available. Visual software named bSpec was created and implemented in order to get automated extraction and classification of the spectral data in a DFBS plate (Figure 1). This software performs all the operations necessary to build the DFBS database and was developed by Giuseppe Cirimele and the MIGG Informatica \& Ricerca team.

A web page and user interface have been created to allow access to the DFBS database for the astronomical community. It can be queried at http://byurakan.phys.uniroma1.it/ or http://arvo.sci.am/ ARVO/DFBS/. The user interface provides an access to general information on the FBS and DFBS, an easy comparison of a spectroscopic DFBS plate with the corresponding direct plates from the DSS1 and DSS2, an access to the database and to the digitized plates. Users may download portions of the plates in FITS format and perform their own analysis. Each spectrum is identified by its coordinates in the USNO-A2 catalogue. Spectra can be downloaded as a single ASCII file containing all the selected spectra or as separate files for each spectrum (a tar or zip archive is actually downloaded). The user interface (the DFBS portal) presently allows the following operations to the guest user: 
- Get Image: Allows users to select a portion of a plate (presently up to $1024 \times 1024$ pixels, i.e. about $\left.26.5^{\prime} \times 26.5^{\prime}\right)$ in FITS format and all spectra of this portion present in the database for downloading (spectra are ASCII files), as well as downloading of the whole selected field.

- Get Spectra: Allows downloading all the spectra in the database within a given distance from a selected central position (cone search); the query may be either interactive, by position, or made by uploading an ASCII file containing one or more positions (Figure 2, right panel). Objects may be selected by $\mathrm{B}, \mathrm{R}$ or $\mathrm{B}-\mathrm{R}$ values. If fewer than 50 objects are selected, the $2 \mathrm{D}$ images can also be downloaded by selecting a check box. The table containing the identifier and the main data for each spectrum can also be downloaded as an ASCII file.
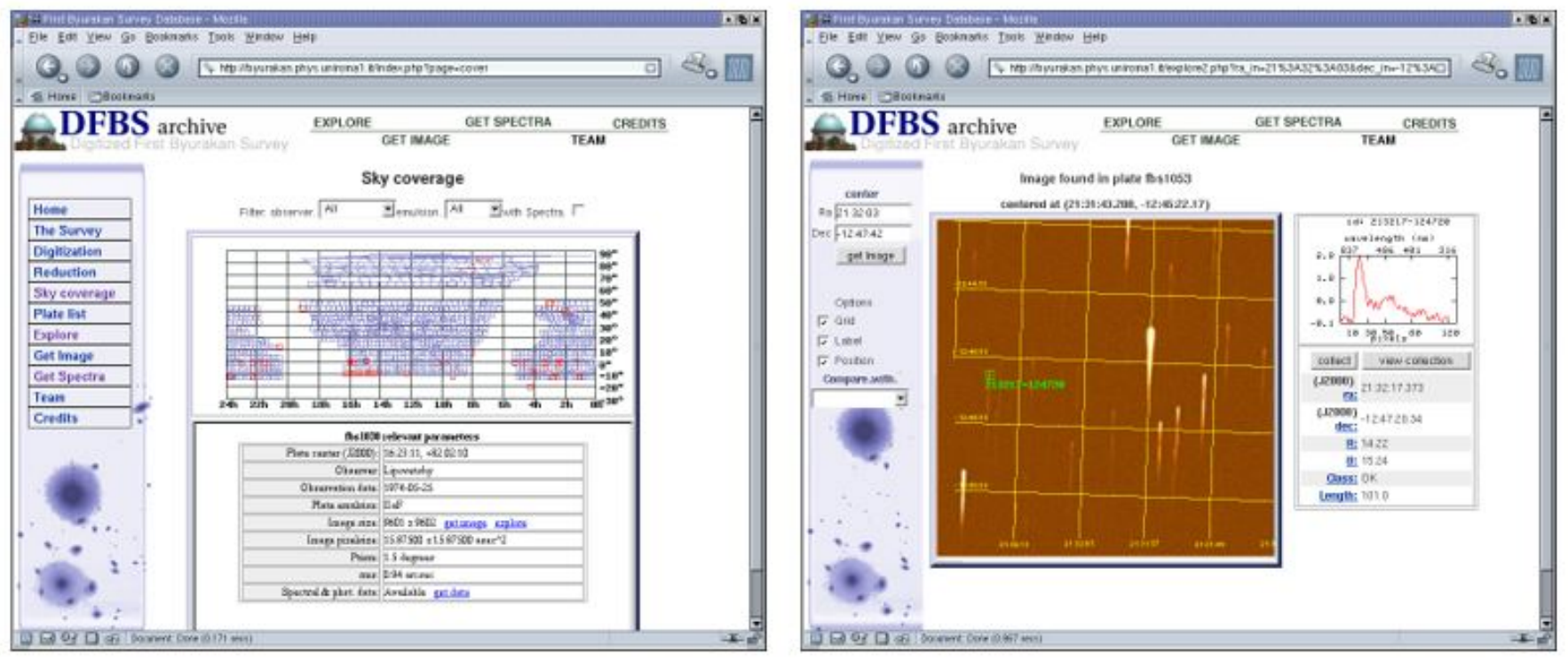

Figure 2. (left) DFBS Sky Coverage and (right) the operation "GETSPECTRA" showing the list of requested spectra and an extraction of a given spectrum on the right.

\section{DFBS based Science Projects}

In Figure 3 we give an example of extraction of an asteroid spectrum from DFBS using VO software SkyBoT proving how useful the DFBS plates can be for follow-up studies (Berthier et al., 2009, Sarkissian et al., 2012, Thuillot et al., 2007). The search for asteroids in DFBS jointly with IMCEE (Observatoire de Paris, France) colleagues was the most advanced research project. Bright $\left(<15^{m}-16^{m}\right)$ asteroids observed in DFBS are being studied, which are divided into "fast" and "slow" ones depending on their motion during the typical DFBS plate exposure time (20 min), more or less than 3". All asteroid spectra are being extracted after they are found by means of SkyBoT. Sample spectra are being modelled similar to Solar spectra. Using these spectra and by means of comparisons with other catalogues, new candidate asteroids are being searched. Spectra analysis of asteroid spectra is being accomplished aimed at obtaining definite physical parameters.

The efficiency of studies of AGB stars based on DFBS low dispersion spectra and follow-up spectroscopy was shown by Nesci et al. (2014b). Gigoyan \& Mickaelian (2007) have found a very high proper motion (PM) M7 type dwarf star, lying about 3 pc from the Sun, FBS $0250+167$. Its PM is 5.050" /yr and it is in the list of the 10 known highest PM stars. Figure 4 shows that only existence of additional observational material, namely DFBS plates from 1969, linked measurements between DSS1 and DSS2 and helped identifying this object and measuring its PM.

The Second Byurakan Survey (SBS; Stepanian 2005) plates are also subject for digitization, as they are hypersensitized and their emulsion is more sensitive for deterioration. 180 plates have been digitized so far. Due to SBS smaller photographic grains, $2400 \mathrm{dpi}(10 \mu \mathrm{m}$ pixel size) is being used and $512 \mathrm{MB}$ files are being obtained for each plate.

Having digitized plates and modern digital observational data, a number of efficient research projects have become possible, such as data discovery, spectral analysis, SED building and fitting, modelling, variability 


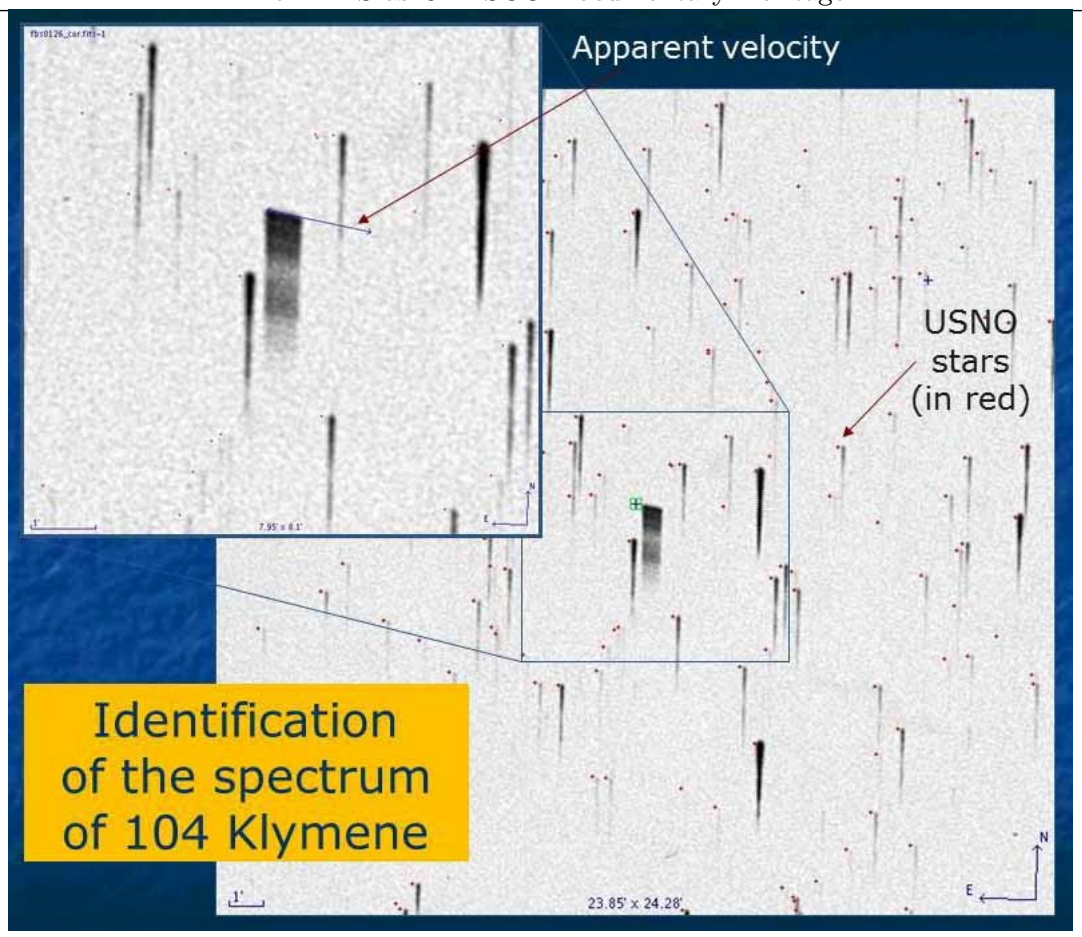

Figure 3. Extraction of an asteroid spectrum from DFBS using VO software SkyBoT.

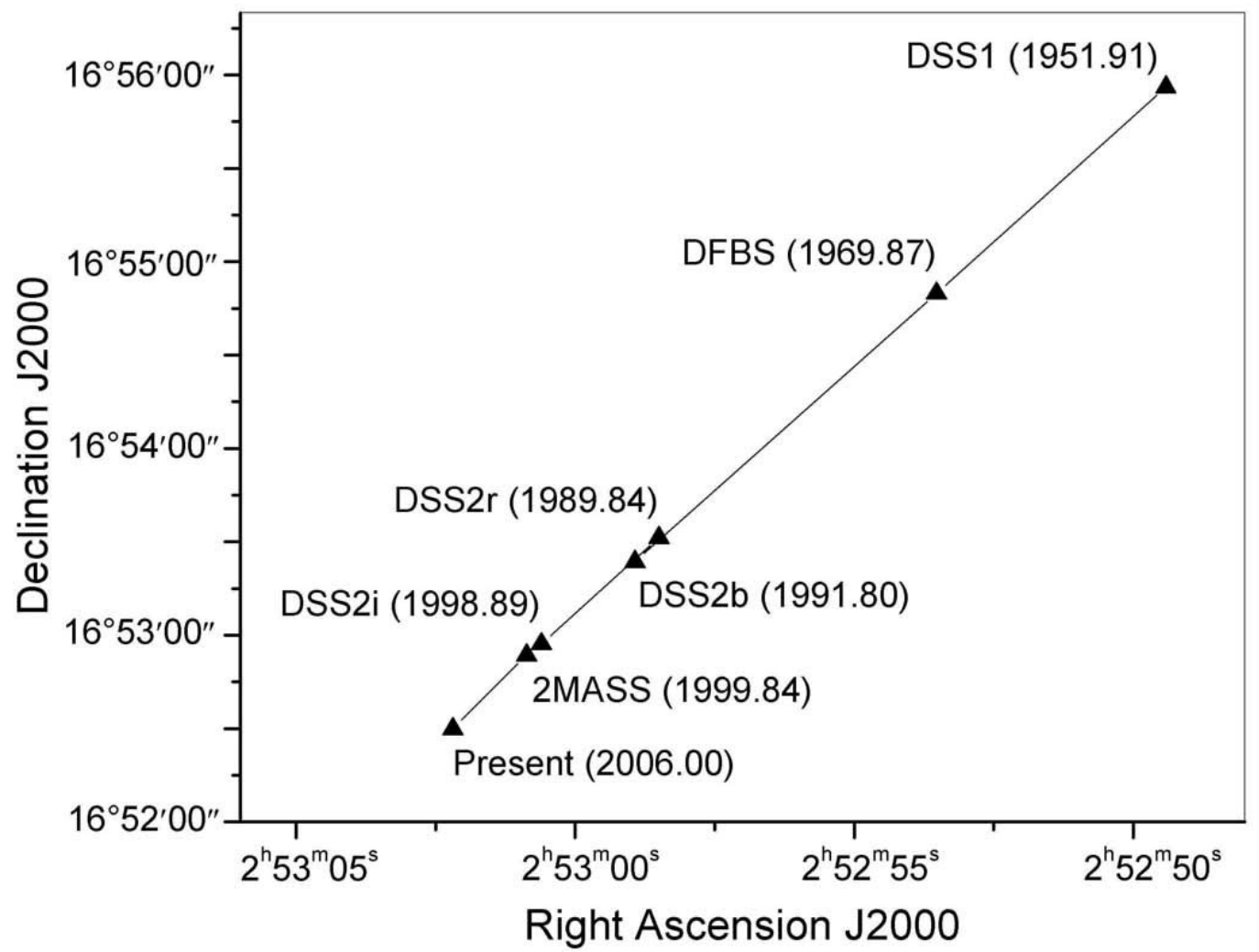

Figure 4. Direction of the motion of the high proper motion $(\mathrm{PM}=5.050$ " $/ \mathrm{yr})$ star FBS $0250+167$, which was found due to DFBS plates.

studies, cross-matching, etc. Some examples are variability studies (Samus \& Antipin, 2012), Cross-matching of Astronomical Catalogs (Malkov, 2012), Search for Asteroids and Exoplanets using VO tools (Sarkissian et al., 2012).

Our science projects are aimed at discoveries of new interesting objects searching definite types of lowdispersion spectra in the DFBS, by optical identifications of non-optical sources (X-ray, IR, radio) also using 
the DFBS and DSS/SDSS, by using cross-correlations of large catalogs and selection of objects by definite criteria, etc.

\section{DFBS as UNESCO "Memory of the World" documentary heritage}

Markarian survey was an outstanding study for all extragalactic (as well as galactic) astronomy; its main features may be given as:

- Markarian survey is the first systematic objective-prism survey in the history of astronomy (later on, objective prism surveys became the main source for search and classification of thousands of objects having some peculiarities and for discovery of many new AGN, white dwarfs, H-alpha emission-line objects (both stars and nebulae), etc.).

- It is the largest objective-prism survey of the Northern sky $\left(17,000 \mathrm{deg}^{2}\right)$, thus providing the largest homogeneous material for unified research.

- It introduced and applied a new method of search for active galaxies, selection by UV-excess.

- Revelation of 1517 UVX galaxies: some 300 AGN and some 1000 HII galaxies.

- Classification of Seyferts into Sy1 and Sy2 types (Weedman \& Khachikyan, 1968).

- Definition of Starburst (SB) galaxies (Weedman, 1977), active galaxies based on high star formation rate.

- Discovery of many new Blue Compact Dwarf Galaxies (BCDG).

- Revelation of 1103 FBS Blue Stellar Objects (BSOs; Mickaelian 2008) and 1471 Late-type Stars (Gigoyan et al., 2019) (2nd Part of the FBS).

- Optical identification of 1577 IRAS sources (samples of Byurakan-IRAS Galaxies (BIG; Mickaelian \& Sargsyan 2004) and Byurakan-IRAS Stars (BIS; Mickaelian \& Gigoyan 2006)); discovery of many new AGN and ULIRGs.

- Markarian survey led to many other objective prism surveys with better spectral resolution and deeper limiting magnitudes, including the Second Byurakan Survey (SBS, Markarian et al. 1983, Stepanian $2005)$.

Markarian galaxies are rather important for various extragalactic studies, such as: Mrk 231 is the most luminous infrared galaxy (ULIRG) in the Local Universe, Mrk 116 is the most metal-deficient blue compact dwarf galaxy (BCDG) (most of the BCDGs are Mrk and SBS galaxies), Mrk 421 and 501 are among the most powerful sources, etc.

Many more UVX and emission-line galaxies have been discovered in similar to Markarian surveys or by other studies. These are Arakelian galaxies having high surface brightness (Arakelian, 1975), Kazarian UVX galaxies (Kazarian et al., 2010), the University of Michigan emission-line galaxies (UM; MacAlpine \& Williams 1981), Case Low-Dispersion Northern Sky Survey galaxies (CG; Pesch et al. 1991), the Montreal blue galaxies (Coziol et al., 1994), SBS UVX and emission-line galaxies (Stepanian, 2005), Kiso UV galaxies (KUG; Miyauchi-Isobe et al. 2010), Hamburg/SAO emission-line galaxies (Pustilnik et al., 2005), GALEX UV-luminous galaxies (Hoopes et al., 2007), etc.

Markarian survey led to the discovery of 1517 UVX galaxies, including some 300 AGN and some 1000 HII galaxies. Classification of Markarian galaxies provided Sy1 and Sy2 types and the definition of Starburst galaxies. Many new BCDG were discovered as well. The continuation of the FBS for stellar objects revealed FBS Blue Stellar Objects and FBS Late-type Stars, as well as optical identifications of IRAS sources have been carried out resulted in discovery of new ULIRGs and AGN. Markarian survey also led to many other objective prism surveys.

Markarian galaxies are reliable objects for MW studies of active galaxies, as they are bright enough and have been detected in all ranges of electromagnetic radiation; from gamma-ray to radio. The Spectral Energy Distribution (SED) provides a possibility to group objects by their shapes and compare to existing physical properties to find various relations and refine the AGN classifications. 
The outstanding scientist Viktor Ambartsumian wrote about Markarian galaxies: "The discovery of Markarian galaxies is a great achievement of our science. Now more and more astronomers both in our country and all over the world seek to study the nature of these objects in more details on the world largest telescopes...". Ambartsumian also compared Markarian survey plates with the treasures of Matenadaran collection of ancient manuscripts.

In 2011, Markarian Survey and its digitized version, DFBS, entered UNESCO's Documentary Heritage "Memory of the World" International Register. It is one of the rarest science treasures that is included in UNESCO lists together with world cultural heritage masterpieces. We give in Figure 5 the related UNESCO Certificate.

The IAU Colloquium \#184 in 2001 was dedicated to Markarian and the IAU Symposium \#304 in 2013 was dedicated to Markarian's $100^{\text {th }}$ anniversary, both related to Markarian Survey and Multiwavelength AGN Surveys in general. The international symposium on "Astronomical Surveys and Big Data" in 2015 was dedicated to the $50^{\text {th }}$ anniversary of Markarian Survey and $10^{\text {th }}$ anniversary of Armenian Virtual Observatory (ArVO), based on DFBS, the digitized Markarian Survey.

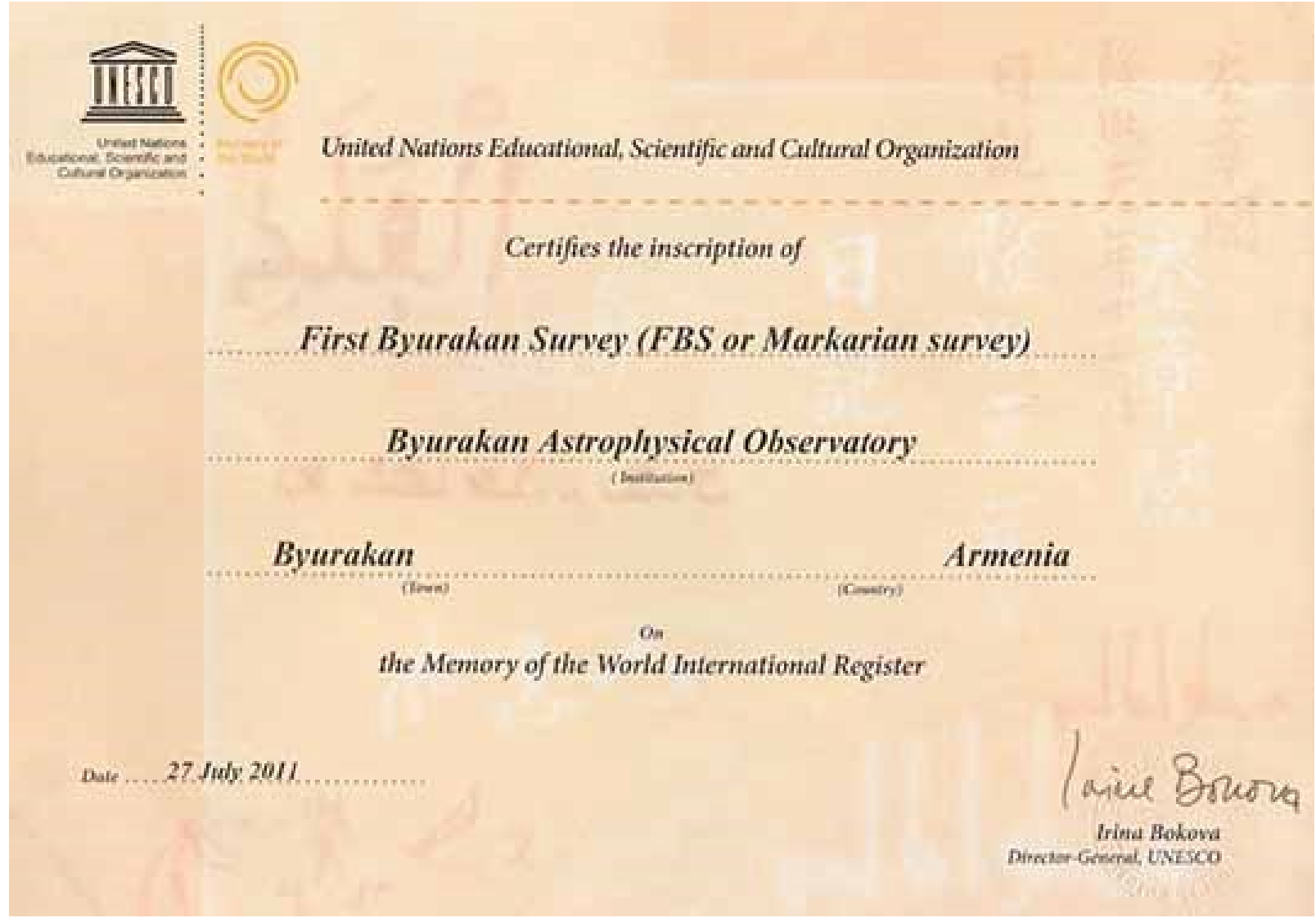

Figure 5. UNESCO certificate awarded for its inscription in the "Memory of the World" documentary heritage International Register

\section{Summary}

There are a number of further possible research projects that will be conducted having the plates digitized:

- Correction of ephemerides of known asteroids and search for new asteroids (ex. Berthier et al. 2009, Thuillot et al. 2007)

- Discovery and study of variable stars (ex. Mickaelian et al. 2011, Nesci et al. 2009)

- Revealing high proper motion stars (ex. Mickaelian \& Sinamyan 2010)

- Study of variability of known blazars and discovery of new blazars 
- Revealing Novae and Supernovae progenitors

- Discovery of new QSOs

- Discovery of new white dwarfs (ex. Sinamyan \& Mickaelian 2011)

- Discovery of new late-type stars (ex. Gigoyan et al. 2019)

- Discovery of optical sources of gamma-ray bursts

- Optical identifications of X-ray, IR and radio sources (ex. Hovhannisyan et al. 2009, Mickaelian \& Gigoyan 2006, Mickaelian \& Sargsyan 2004, Mickaelian et al. 2006c).

\section{Acknowledgements}

The work was supported by the Science Committee of RA, in the frames of the research project No. 21AG-1C053 "Revelation of the early stages of galaxy evolution by means of multiwavelength study of active galaxies".

\section{References}

Arakelian M. A., 1975, Communications of the Byurakan Astrophysical Observatory, 47, 3

Berthier J., Sarkissian A., Mickaelian A., Thuillot W., 2009, in European Planetary Science Congress 2009. p. 526

Coziol R., Demers S., Pena M., Barneoud R., 1994, Astron. J. , 108, 405

Erastova L. K., 2004, Astrophysics, 47, 79

Gigoyan K. S., Mickaelian A. M., 2007, Astrophysics, 50, 54

Gigoyan K. S., Mickaelian A. M., 2012, Mon. Not. R. Astron. Soc. , 419, 3346

Gigoyan K. S., Abrahamyan H. V., Azzopardi M., Mauron N., Russeil D., Sinamyan P., 2003, Astrophysics, 46, 475

Gigoyan K. S., Sinamyan P. K., Engels D., Mickaelian A. M., 2010, Astrophysics, 53, 123

Gigoyan K. S., Mickaelian A. M., Kostandyan G. R., 2019, Mon. Not. R. Astron. Soc. , 489, 2030

Hoopes C. G., et al., 2007, Astrophys. J. Suppl. Ser., 173, 441

Hovhannisyan L. R., et al., 2009, Astron. J. , 138, 251

Hudec R., 2014, in Astroplate 2014. p. 1

Kazantseva L., 2014, in Astroplate 2014. p. 13

Kazarian M. A., Adibekyan V. Z., McLean B., Allen R. J., Petrosian A. R., 2010, Astrophysics, 53, 57

Knyazyan A., Mickaelian A., Astsatryan H., 2011, International Journal "Information Theories and Applications, 18, 243

Knyazyan A. V., Astsatryan H. V., Mickaelian A. M., 2016, in Mickaelian A., Lawrence A., Magakian T., eds, Astronomical Society of the Pacific Conference Series Vol. 505, Astronomical Surveys and Big Data. p. 236

MacAlpine G. M., Williams G. A., 1981, Astrophys. J. Suppl. Ser. , 45, 113

Malkov O. Y., 2012, in Mickaelian A. M., Malkov O. Y., Samus N. N., eds, Fifty years of Cosmic Era: Real and Virtual Studies of the Sky. Conference of Young Scientists of CIS Countries. pp 48-55

Markarian B. E., Lipovetskii V. A., Stepanian D. A., 1983, Astrofizika, 19, 29

Markarian B. E., Lipovetsky V. A., Stepanian J. A., Erastova L. K., Shapovalova A. I., 1989, Soobshcheniya Spetsial'noj Astrofizicheskoj Observatorii, 62,5

Massaro E., Mickaelian A. M., Nesci R., Weedman D., 2008, The Digitized First Byurakan Survey. ARACNE Editrice, Rome, 78p.

Mazzarella J. M., Balzano V. A., 1986, Astrophys. J. Suppl. Ser. , 62, 751

Mickaelian A. M., 2004, SCAN-IT No. 2, 2, 16

Mickaelian A. M., 2006, Romanian Astronomical Journal, 16, 23

Mickaelian A. M., 2007, Highlights of Astronomy, 14, 594

Mickaelian A. M., 2008, Astron. J., 136, 946

Mickaelian A. M., 2012, in Mickaelian A. M., Malkov O. Y., Samus N. N., eds, Fifty years of Cosmic Era: Real and Virtual Studies of the Sky. Conference of Young Scientists of CIS Countries. pp 29-38 
Mickaelian A., 2014a, in Astroplate 2014. p. 109

Mickaelian A. M., 2014b, in Mickaelian A. M., Sanders D. B., eds, Vol. 304, Multiwavelength AGN Surveys and Studies. pp 1-10, doi:10.1017/S1743921314003147

Mickaelian A. M., 2016, in Mickaelian A., Lawrence A., Magakian T., eds, Astronomical Society of the Pacific Conference Series Vol. 505, Astronomical Surveys and Big Data. p. 3

Mickaelian A. M., Gigoyan K. S., 2006, Astron. Astrophys. , 455, 765

Mickaelian A. M., Sargsyan L. A., 2004, Astrophysics, 47, 91

Mickaelian A. M., Sinamyan P. K., 2010, Mon. Not. R. Astron. Soc. , 407, 681

Mickaelian A. M., Hagen H. J., Sargsyan L. A., Mikayelyan G. A., 2005a, VizieR Online Data Catalog, p. VI/116

Mickaelian A. M., et al., 2005b, in Colless M., Staveley-Smith L., Stathakis R. A., eds, Vol. 216, Maps of the Cosmos. p. 230, doi:10.1017/S0074180900196676

Mickaelian A. M., Sargsyan L. A., Mikayelyan G. A., Erastova L. K., Sinamian P. K., 2006a, in Tsvetkov M., Golev V., Murtagh F., Molina R., eds, Virtual Observatory: Plate Content Digitization, Archive Mining and Image Sequence Processing. pp 82-92

Mickaelian A. M., Gigoyan K. S., Nesci R., Rossi C., 2006b, Mem.S.A.It., 77, 1159

Mickaelian A. M., Hovhannisyan L. R., Engels D., Hagen H. J., Voges W., 2006c, Astron. Astrophys. , 449, 425

Mickaelian A. M., et al., 2007, Astron. Astrophys. , 464, 1177

Mickaelian A. M., et al., 2008a, in Guainazzi M., Osuna P., eds, Astronomical Spectroscopy and Virtual Observatory. p. 29

Mickaelian A. M., et al., 2008b, Romanian Astronomical Journal, 18, 249

Mickaelian A. M., et al., 2009a, Proceedings of CSIT-2009 conference, 7, 420

Mickaelian A., et al., 2009b, CSIT 2009 Proceedings, 7, 424

Mickaelian A. M., Sargsyan L. A., Astsatryan H. V., Cirimele G., Nesci R., 2009c, Defense Science Journal, 8, 152

Mickaelian A. M., Sargsyan L. A., Nesci R., Cirimele G., Sarkissian A., 2010, in Mizumoto Y., Morita K. I., Ohishi M., eds, Astronomical Society of the Pacific Conference Series Vol. 434, Astronomical Data Analysis Software and Systems XIX. p. 325

Mickaelian A. M., Mikayelyan G. A., Sinamyan P. K., 2011, Mon. Not. R. Astron. Soc. , 415, 1061

Mickaelian A. M., Malkov O. Y., Samus N. N., eds, 2012, Fifty years of Cosmic Era: Real and Virtual Studies of the Sky

Mickaelian A. M., et al., 2016a, in Mickaelian A., Lawrence A., Magakian T., eds, Astronomical Society of the Pacific Conference Series Vol. 505, Astronomical Surveys and Big Data. p. 16

Mickaelian A. M., et al., 2016b, in Mickaelian A., Lawrence A., Magakian T., eds, Astronomical Society of the Pacific Conference Series Vol. 505, Astronomical Surveys and Big Data. pp 262-273

Mickaelian A. M., Sarkissian A., Berthier J., Meftah M., Thuillot W., Vachier F., 2019, Icarus, 330, 5

Miyauchi-Isobe N., Maehara H., Nakajima K., 2010, Publications of the National Astronomical Observatory of Japan, 13, 9

Nesci R., Rossi C., Sclavi S., Gaudenzi S., Mickaelian A. M., Sargsyan L. A., Erastova L. K., 2004, Baltic Astronomy, 13,671

Nesci R., Mickaelian A., Rossi C., 2009, The Astronomer's Telegram, 2338, 1

Nesci R., Bagaglia M., Nucciarelli G., 2014a, in Astroplate 2014. p. 75

Nesci R., Gaudenzi S., Rossi C., Pezzotti C., Gigoyan K., Mauron N., 2014b, in Astroplate 2014. p. 91 (arXiv:1404.3842)

Osborn W., 2014, in Astroplate 2014. p. 15

Pesch P., Sanduleak N., Stephenson C. B., 1991, Astrophys. J. Suppl. Ser. , 76, 1043

Pustilnik S. A., et al., 2005, Astron. Astrophys. , 442, 109

Samus N. N., Antipin S., 2012, in Mickaelian A. M., Malkov O. Y., Samus N. N., eds, Fifty years of Cosmic Era: Real and Virtual Studies of the Sky. Conference of Young Scientists of CIS Countries. pp 39-47

Sarkissian A., Arzoumanian E., Mickaelian A. M., Berthier J., Thuillot W., Vachier F., 2012, in Mickaelian A. M., Malkov O. Y., Samus N. N., eds, Fifty years of Cosmic Era: Real and Virtual Studies of the Sky. Conference of Young Scientists of CIS Countries. pp 56-64

Sinamyan P. K., Mickaelian A. M., 2009, Astrophysics, 52, 76

Sinamyan P. K., Mickaelian A. M., 2011, Astrophysics, 54, 403

Sokolovsky K. V., Antipin S. V., Zubareva A. M., Kolesnikova D. M., Lebedev A. A., Samus' N. N., Sat L. A., 2014, Astronomy Reports, 58,319 
Stupka J., Benesova E., 2014, in Astroplate 2014. p. 31

Thuillot W., et al., 2007, Highlights of Astronomy, 14, 616

Tsvetkov M., Tsvetkova K., 2012, in Griffin E., Hanisch R., Seaman R., eds, Vol. 285, New Horizons in Time Domain Astronomy. pp 417-419, doi:10.1017/S1743921312001275

Véron-Cetty M. P., Véron P., 2010, Astron. Astrophys. , 518, A10

Weedman D. W., 1977, Vistas in Astronomy, 21, 55

Weedman D. W., Khachikyan E. E., 1968, Astrophysics, 4, 243 